\title{
The Role and Significance of Wnt5a in Regulating Epithelial-Mesenchymal Transition in Endometrioid Adenocarcinoma
}

\author{
Ningning Yang $\mathbb{D}^{1,2}$ \\ Hongchun Chen $\mathbb{D}^{1,2}$ \\ Yuchen Huang $\mathbb{D}^{1,2}$ \\ Xuexue Song $\mathbb{1}^{3}$ \\ Panpan Yang (1) 4 \\ Shan Zhang $\mathbb{D}^{5}$ \\ Wentian Yan $\mathbb{D}^{1,2}$ \\ Nan Li $\mathbb{D}^{1,2}$ \\ Zhenzhong Feng $\mathbb{D}^{1,2}$
}

'Department of Pathology, First Affiliated Hospital of Bengbu Medical College, Bengbu, 233000, People's Republic of China; ${ }^{2}$ Department of Pathology, Bengbu Medical College, Bengbu, 233000,

People's Republic of China; ${ }^{3}$ Department of Pathology, First Affiliated Hospital of University of Science and Technology of China, Hefei, 230000, People's Republic of China; ${ }^{4}$ Department of Pathology, Second Affiliated Hospital of Anhui Medical University, Hefei, 230000, People's Republic of China; ${ }^{5}$ Department of Pathology, Second People's Hospital of Hefei, Hefei, 230000, People's Republic of China
Purpose: As a non-classical ligand of Wnt, the abnormal regulation of Wnt5a contributes to the progression of malignant tumors; however, its effects differ depending on tumor type. Here, we evaluated the expression and significance of Wnt5a in endometrioid adenocarcinoma and its relationship with epithelial-mesenchymal transition (EMT)-related proteins.

Patients and Methods: Immunohistochemical streptavidin-peroxidase method and reverse transcription polymerase chain reaction (RT-PCR) method were used to analyze the expression and correlation of Wnt5a, and EMT-related protein $\beta$-catenin, E-cadherin and enhancer of zeste homolog 2 (EZH2) in endometrial cancer tissues and cell samples of each group.

Results: The expression of Wnt5a and E-cadherin decreased in the following order, normal endometrium $>$ atypical hyperplasia endometrium $>$ endometrioid adenocarcinoma. In contrast, the expression of $\beta$-catenin and EZH2 increased gradually. Moreover, Wnt5a expression was associated with the degree of tissue differentiation, International Federation of Gynecology and Obstetrics (FIGO) stage, and lymph node metastasis (all $P<0.05$ ). Wnt5a expression was also negatively correlated with $\beta$-catenin and EZH2 expression and positively correlated with E-cadherin expression. RT-PCR results further indicated that E-cadherin mRNA expression was upregulated in a Wnt5a-overexpressing Ishikawa cell line compared to cells transfected with an empty vector or negative control cells $(P<0.01)$. Furthermore, the expression of EZH2 and $\beta$-catenin mRNA was downregulated in overexpressing cells compared to empty vector and negative control cells $(P<0.01)$.

Conclusion: Wnt5a may elicit a suppressive effect on endometrioid adenocarcinoma by inhibiting EMT. This study provides a theoretical basis for the pathological diagnosis and targeted therapy of endometrioid adenocarcinoma and extends our understanding of the Wnt5a signaling pathway.

Keywords: endometrioid adenocarcinoma, Wnt5a, epithelial-mesenchymal transition, immunohistochemistry

\section{Introduction}

Endometrial carcinoma (EC), a malignant tumor that threatens the reproductive health of women, ${ }^{1}$ accounts for $20-30 \%$ of all female reproductive tract tumors. Endometrioid adenocarcinoma (EA), also known as type I EC, is an estrogen-dependent tumor with a good prognosis and is the most common subtype of endometrial epithelial tumors, accounting for $80-85 \%$ of EC cases. The occurrence and development of EC are attributed to various abnormal interactions among signal transduction pathways and
Correspondence: Zhenzhong Feng; Nan Li Department of Pathology, First Affiliated Hospital of Bengbu Medical College,

No. 287 Changhuai Road, Bengbu, Anhui Province, People's Republic of China Tel +86 I5055289508

Email fzz18297301626@163.com;

linanangelI00@sina.com 
genes. However, the specific pathogenesis of EC remains unknown, and studies on oncogenes and tumor suppressor genes are a major research topic in the field of EA. Genetic factors associated with endometrioid carcinoma include K-RAS mutations, PTEN inactivation, and $\beta$-catenin activation. Abnormal signal transduction of the $\mathrm{Wnt} / \beta$-catenin pathway resulting from $\beta$-catenin activation is a particularly important factor in EA. ${ }^{2}$

Wnt proteins are secreted glycoproteins widely expressed in various tissues and show high homology with Wingless in Drosophila melanogaster. The Wnt-mediated signaling pathway regulates cell growth, differentiation, proliferation, apoptosis, and migration, which are all closely associated with invasion and metastasis in various malignant tumors. Wnt5a, a member of the Wnt family, was first discovered in a mouse mammary gland by Clark et $\mathrm{al}^{3}$ and is located on the chromosome region 3p14.2-p21.1 in humans. The Wnt5a gene encodes a cysteine-rich growth factor and participates in signal transmission between cells during growth and differentiation. Wnt5a is an evolutionarily conserved non-classical Wnt ligand ${ }^{4}$ that not only activates the non-classical $\mathrm{Wnt} / \mathrm{Ca}^{2+}$ pathway but also regulates the classical $\mathrm{Wnt} / \beta$ catenin pathway. Wnt5a binds to tyrosine kinase-like orphan receptors, inhibits the $\mathrm{Wnt} / \beta$-catenin pathway, and interacts with Frizzled and low-density lipoprotein receptor-related transmembrane proteins to activate the $\mathrm{Wnt} / \beta$-catenin pathway. ${ }^{5}$ Wnt5a expression differs among tissues, and while it has been associated with carcinogenesis in nonsmall cell lung cancer, stomach cancer, prostatic cancer, and melanoma of the skin, an inhibitory role in liver cancer, thyroid cancer, and hematological tumors has also been reported. ${ }^{6}$ The Wnt signaling pathway can also induce the epithelial-mesenchymal transition (EMT) in tumor cells, thereby promoting tumor invasion and metastasis. ${ }^{7}$ Meanwhile, few studies have examined the expression of Wnt5a in EA and its correlation with EMT-related proteins. In this study, the expression patterns of Wnt5a and the EMTinducing factors $\beta$-catenin, E-cadherin, and enhancer of zeste homolog 2 (EZH2) were analyzed in EA to explore the role and significance of Wnt5a in the occurrence and development of EA. This study provides a theoretical basis for the diagnosis and treatment of EA.

\section{Materials and Methods}

\section{Clinical Data}

From January 2011 to December 2017, 132 human endometrial tissue samples comprising 10 normal endometrium samples, 15 atypical hyperplasia endometrium samples, and 107 EA samples were collected. The following patient inclusion criteria were applied: (1) complete clinicopathological data, (2) all samples were surgically removed, and (3) patients had not received radiotherapy, chemotherapy, or immunotherapy prior to surgery. Cases were restaged according to the 2018 International Federation of Obstetrics and Gynecology (FIGO) staging standard. In total, 65 cases were classified as stage I, 12 as stage II, and 30 as stages III-IV. According to the histological classification of endometrioid carcinoma, 30 cases were in the G1 stage, 45 in the G2 stage, and 32 in the G3 stage. There were 61 cases without muscle layer infiltration $(\leq 1 /$ $2)$ and 46 with muscle layer infiltration $(>1 / 2)$. There were also 29 cases with lymph node metastasis and 78 without lymph node metastasis. Informed consent was obtained from all patients and their families. The study was approved by the Ethics Committee of the First Affiliated Hospital of Bengbu Medical College (NO. 2020057). This study was conducted in accordance with the Helsinki Declaration.

\section{Immunohistochemistry and Determination of Staining Profiles}

All samples were fixed with $10 \%$ neutral formalin, dehydrated, embedded in paraffin, and cut into $4-\mu \mathrm{m}$-thick slices. After dewaxing with xylene and rehydrating with alcohol at various concentrations, the sections were soaked in 3\% hydrogen peroxide for $15 \mathrm{~min}$. After washing with distilled water, sections were soaked in a citric acid buffer in a boiling pressure cooker for $2 \mathrm{~min}$. The sections were washed several times with running water, distilled water, and phosphate-buffered saline (PBS) prior to sealing and antigen heat repair. Primary antibodies against Wnt5a (ab229200, Abcam, Cambridge, UK), EZH2 (ab186006, Abcam), $\beta$-catenin (A19657, ABclonal, Wuhan, China), and E-cadherin (A3044, ABclonal) were diluted with PBS to $1: 200,1: 400,1: 200$, and 1:200, respectively. The sections were incubated with the relevant antibodies at $37{ }^{\circ} \mathrm{C}$ for 30 min. Diaminobenzidine (DAB) was added for color development, and hematoxylin was used for counterstaining, followed by neutral gum for sealing. PBS was used as a negative control. To delineate false positives and false negatives, known breast cancer-positive tissues were used as positive controls. Except for the primary antibody, all the reagents used in the immunohistochemical experiment were purchased from Fuzhou Maixin Biological Co. Ltd. 
Immunohistochemical results were reviewed by two senior pathologists. Positive signals for Wnt5a, EZH2, and E-cadherin were localized in the cytoplasm, nuclei, and cell membranes, respectively. Positive $\beta$-catenin signals were localized in the cell membrane of the normal endometrial glandular epithelium, endometrium with atypical hyperplasia, and nuclei of EA cells. The cell staining intensity and staining range of Wnt5a were evaluated and scored as follows: colorless, 0 points; light yellow, 1 point; claybank, 2 points; and tan, 3 points. The staining point-range was determined based on the proportion of positive cells. Five high-power microscope fields were randomly selected to calculate the percentage of positive cells, and the mean value was determined. If the number of positive cells was $<5 \%, 0$ points were assigned, 1 point was assigned for 5 $25 \%, 2$ points for $26-50 \%, 3$ points for $51-75 \%$, and 4 points for $>75 \%$. The comprehensive score was the product of the dyeing intensity and dyeing range. When the comprehensive score was $<3$, it was considered negative, and when $>3$, it was considered positive.

\section{Cell Culture and Transfection}

The human EC Ishikawa cell (Nanjing Kaiji Biological Co., Ltd., China) line was cultured in RPMI-1640 medium (Servicebio, Wuhan, China) containing 10\% fetal bovine serum (Sijiqin, Suzhou, China) and 1\% penicillin-streptomycin solution (Biosharp, Hefei, China) in an incubator at $37{ }^{\circ} \mathrm{C}$ and $5 \% \mathrm{CO}_{2}$. On the day before transfection, the cells to be transfected were inoculated in a T25 standard culture flask (the number of cells was about $2 \times 10^{6}$ ) and cultured in an antibiotic-free environment for 18-24 $\mathrm{h}$ until the confluence reached about $70-90 \%$. Every bottle of cells was mixed with $1.5 \mathrm{~mL}$ opti-MEM (ThermoFisher, NewYork, USA); additionally, $7.5 \mu \mathrm{g}$ plasmid DNA (nm003392) (JiMa gene, Suzhou, China) and $15 \mu \mathrm{L}$ Lipo 2000 (ThermoFisher) were mixed with $1.5 \mathrm{~mL}$ opti-MEM, and the flask was then left at $25{ }^{\circ} \mathrm{C}$ for $20 \mathrm{~min}$. The old culture medium was removed, the cells were washed twice with PBS, and the transfection complex was added to the bottom of the culture flask drop by drop. Finally, opti-MEM was added until the liquid in the culture flask reached $6 \mathrm{~mL}$, and the flask was placed in a $5 \% \mathrm{CO}_{2}$ incubator at $37{ }^{\circ} \mathrm{C}$ for $5 \mathrm{~h}$. The medium was later changed to an antibiotic-free culture medium to continue culturing. Subsequent experiments were carried out $48 \mathrm{~h}$ after successful transfection.

\section{Quantitative Reverse Transcription Polymerase Chain Reaction (RT-PCR)}

Total RNA was extracted from cells of tissue samples with TRIzol (ThermoFisher). RNA purity and concentration were detected using a Thermo Scientific full-wavelength scanning multifunctional reader. cDNA was synthesized with the reverse transcription kit (ThermoFisher). The following primers (JiMa gene) were used for PCR: WNT5A forward: 5'ACGGCTTCCCAATAACAGTAGC-3', reverse: 5'CAACCCAACACGCATTTCA-3'; EZH2 forward: 5'ACGGCTTCCCAATAACAGTAG-3', reverse: 5'TTGACACCGAGAATTTGCTTC-3'; CDH1 (E-cadherin) forward: 5'-CTTGGTCTACGCCTGGGACT-3', reverse: 5'TGTGAGCAATTCTGCTTGGA-3'; CTNNB1 ( $\beta$-catenin) forward: 5'-CTGCCAAGTGGGTGGTATAGAG, reverse: 5'-CGGGACAAAGGGCAAGAT-3'; GAPDH forward: 5'CATGAGAAGTATGACAACAGCCT-3', reverse: 5'-AGT CCTTCCACGATACCAAAGT-3'. The product fragment lengths were 74(bp), 178(bp), 251(bp), 130(bp), and 113 (bp), respectively.

\section{Follow-Up}

All 107 EA cases were followed up retrospectively for 2496 months until December 2019. Patients who were disease-free and those lost at the follow-up deadline were designated as a truncation, and patients who died of EA were designated as a defined event.

\section{Statistical Analysis}

All statistical analyses were performed using SPSS 21.0 software for Windows (IBM, New York, USA). All $\mathrm{P}$-values were two-sided, and $\mathrm{P}<0.05$ were considered statistically significant. Chi-square tests were performed to analyze the difference between Wnt5a-positive and -negative groups with respect to clinicopathological parameters and the expression of $\beta$-catenin, E-cadherin, and EZH2. Statistical analyses were also performed to detect differences in expression of the four proteins between the overexpressing and empty vector groups and negative control cells. The survival of patients with endometrioid carcinoma was analyzed using the Kaplan-Meier method.

\section{Results}

\section{Expression of Wnt5a and EMT-Related} Proteins in Endometrial Tissues

The expression of Wnt5a and E-cadherin, determined by immunohistochemistry, were downregulated in the EA 
tissue samples compared to the atypical hyperplasia and normal endometrium samples. In contrast, expression levels of $\beta$-catenin and EZH2 were upregulated in the EA samples, compared to atypical hyperplasia and normal endometrium (Table 1, Figure 1).

\section{Relationship Between Wnt5a Expression and the Clinicopathological Features of}

\section{Endometrioid Adenocarcinomas}

In the 107 cases of EA, the positivity rate of Wnt5a in the poorly differentiated group was $15.6 \%$, which was significantly lower than that in the well-differentiated (46.7\%) and moderately differentiated $(42.2 \%)$ groups. The positivity rate of Wnt5a in stage III-IV samples was $16.7 \%$, which was lower than that in stage I and II samples (47.7\%, $P=0.011$ ). The positivity rate of Wnt5a in patients with metastasis $(17.2 \%)$ was significantly lower than in patients without metastasis $(42.3 \%, P=0.018)$. Finally, Wnt5a expression in EA was not associated with patient age or extent of myometrial invasion $(P>0.05)$; however, it was associated with the degree of tumor differentiation, pathological stage, and lymph node metastasis $(P<0.05)$ (Table 2).

\section{Relationship Between Wnt5a Expression and EMT-Related Indicators in \\ Endometrioid Adenocarcinoma}

Of the 22 negative cases for $\beta$-catenin expression, 13 were positive for Wnt5a expression, ie, 59.1\% of Wnt5a-positive cases exhibited a loss of $\beta$-catenin expression. Moreover, eighteen of the 34 cases that were positive for E-cadherin expression (52.9\%) were positive for Wnt5a expression, whereas 17 of 32 EZH2-negative cases were Wnt5a-positive (53.1\%). Spearman correlation analysis further showed that Wnt5a expression significantly correlated with these three EMT-related proteins in the EA group $(P<0.05)$ (Table 3, Figure 1).

\section{Quantitative RT-PCR Test results}

The mean \pm standard deviation of the relative quantification (RQ) value was assessed with the SPSS 21.0 software. The samples were analyzed in triplicates. The RQ values of WNT5A, EZH2, CDH1, and CTNNB1 mRNA were 1.61 $\pm 0.09,0.49 \pm 0.16,3.4 \pm 0.28$, and $0.5 \pm 0.10$, respectively, in the overexpression group. In the empty carrier group they were $0.85 \pm 0.14,0.93 \pm 0.09,0.82 \pm 0.21$, and $0.90 \pm 0.19$, respectively; in the negative control group all were $1 \pm 0$. These results indicate that the mRNA expression of WNT5A and $C D H 1$ was higher than that of the empty vector group and negative control group $(P<0.01$ for each). Meanwhile, $E Z H 2$ and CTNNB1 were downregulated in the overexpressing cells compared to the empty vector and negative control cells ( $P<0.01$ for each). In addition, no significant difference was observed in the expression of WNT5A, EZH2, CDH1, and $C T N N B 1$ between the empty vector and negative control groups (Figure 2).

\section{Follow-Up}

Kaplan-Meier survival analysis showed that the average survival time of Wnt5a-negative patients was 63 months and the median survival was 61 months. The average survival time of Wnt5a positive patients was 77 months and the median survival time was 55 months. The difference in prognosis between the groups was significant $\left(\chi^{2}=\right.$ $10.773, P<0.01$ ) (Figure 3).

\section{Discussion}

Studies have indicated that Wnt5a can affect cell migration, invasion, and angiogenesis and participates in tumor development via regulating multiple functions. Kurayoshi et $\mathrm{al}^{8}$ reported that Wnt5a is highly expressed in gastric cancer tissues and is related to invasiveness and poor prognosis. Therefore, this protein is a good prognostic indicator and an effective molecular target for gastric cancer treatment. Kremenevskaja et $\mathrm{al}^{9}$ also found that mRNA expression levels of Wnt5a in thyroid cancer tissues are substantially higher than in normal tissues,

Table I Expression of Wnt5a and EMT-Related Proteins in Endometrial Tissues

\begin{tabular}{|c|c|c|c|c|c|c|c|c|c|c|c|c|c|}
\hline \multirow[t]{2}{*}{ Group } & \multirow[t]{2}{*}{ Total } & \multicolumn{3}{|c|}{ Wnt5a } & \multicolumn{3}{|c|}{$\beta$-catenin } & \multicolumn{3}{|c|}{ E-cadherin } & \multicolumn{3}{|c|}{ EZH2 } \\
\hline & & - & + & $\mathbf{P}$ & - & + & $\mathbf{P}$ & - & + & $\mathbf{P}$ & - & + & $\mathbf{P}$ \\
\hline Normal endometrium & 10 & 2 & 8 & & 8 & 2 & & 1 & 9 & & 8 & 2 & \\
\hline Atypical hyperplasia of endometrium & 15 & 4 & II & & 11 & 4 & & 7 & 8 & & 6 & 9 & \\
\hline Endometrioid adenocarcinoma & 107 & 69 & 38 & $<0.01$ & 22 & 85 & $<0.01$ & 73 & 34 & $<0.01$ & 32 & 75 & $<0.01$ \\
\hline
\end{tabular}

Abbreviations: EZH2, enhancer of zeste homolog 2; P, P-value. 


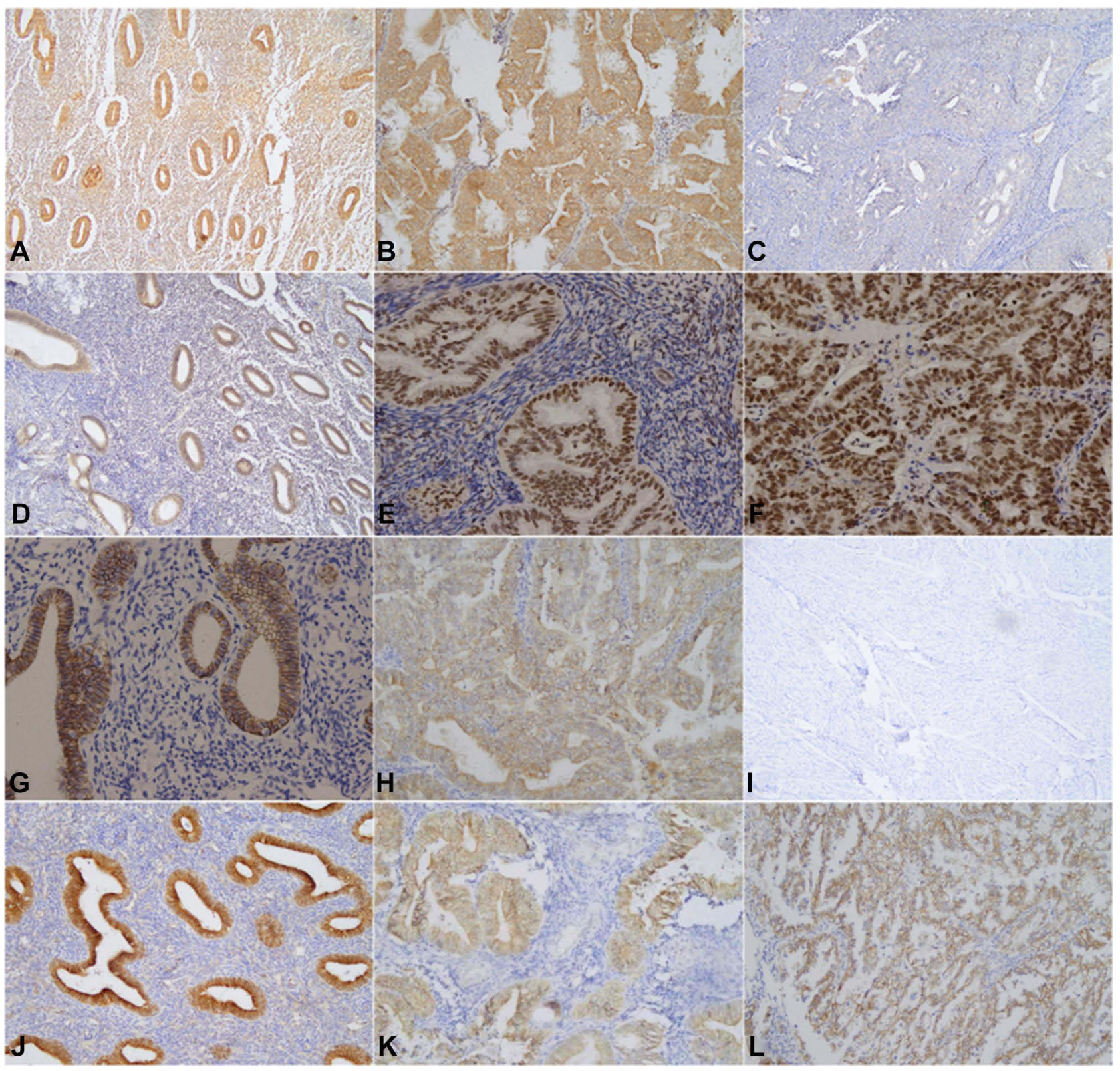

Figure I Immunohistochemical staining of normal endometrial tissue, atypical hyperplasia endometrial tissue and endometrial tumor tissue was carried out using thestreptavidin-peroxidase method. Normal endometrial tissue: Figure (A)Wnt5a(+), Figure (D) EZH2(-); Figure (G) E-cadherin(+), Figure (J) $\beta$-catenin(-). Atypical hyperplasia of endometrial tissue: Figure $(\mathbf{B}) \mathrm{Wnt5a}(+)$, Figure $(\mathbf{E})$ EZH2(+), Figure $(\mathbf{H})$ E-cadherin $(+)$, Figure $(\mathbf{K}) \beta$-catenin $(+)$. Endometrial carcinoma tissue: Figure $(\mathbf{C})$ Wnt5a(-), Figure (F) EZH2(+), Figure (I) E-cadherin (-), and Figure (L) $\beta$-catenin $(+)$.

whereas Wnt5a expression in normal thyroid tissues and anaplastic tumor tissues is low. It is worth mentioning that Wnt5a plays a different role in breast cancer and colon cancer. Wnt5a plays an oncogenic role in non-immortal breast cancer but plays an anti-oncogenic role in invasive breast ductal cancer. ${ }^{10}$ Regarding colon cancer, some people have reported that it is positively correlated with a good prognosis, ${ }^{11}$ while others have reported that it promotes colon cancer progression and indicates poor prognosis. ${ }^{12}$ Interestingly, our results showed that Wnt5a expression was at basal levels in the normal endometrium (80\%) but gradually decreased in the endometrium with atypical hyperplasia (73.3\%) and in EA cases (35.5\%). In EA, Wnt5a expression is closely associated with tumor invasion depth, tissue differentiation, pathological stage, and degree of metastasis. These results suggest that Wnt5a plays an anti-cancer role in EA, possibly via the activation of the non-classical Wnt signaling pathway.

EMT refers to a process in which epithelial cells gradually lose their epithelial characteristics and acquire stromal 
Table 2 Relationship Between Wnt5a Expression and Clinicopathological Features in Endometrioid Adenocarcinoma

\begin{tabular}{|c|c|c|c|c|c|}
\hline Clinicopathological Parameters & Total $/ \mathbf{n}$ & Wnt5a (-) (\%) & Wnt5a (+) (\%) & $\chi^{2}$ value & P-value \\
\hline \multicolumn{6}{|l|}{ Age (Years) } \\
\hline$<50$ & 32 & $21(65.6)$ & II (34.4) & & \\
\hline$\geq 50$ & 75 & $48(64.0)$ & $27(36.0)$ & 0.026 & 1.000 \\
\hline \multicolumn{6}{|l|}{ Infiltration depth } \\
\hline$\leq \mathrm{I} / 2$ muscle layer & 61 & $40(65.6)$ & $21(34.4)$ & & \\
\hline$>1 / 2$ muscle layer & 46 & $29(63.0)$ & $17(37.0)$ & 0.073 & 0.787 \\
\hline \multicolumn{6}{|l|}{ Degree of Tissue Differentiation } \\
\hline GI & 30 & $16(53.3)$ & $14(46.7)$ & & \\
\hline G2 & 45 & $26(57.8)$ & $19(42.2)$ & & \\
\hline G3 & 32 & $27(84.4)$ & $5(15.6)$ & 8.041 & 0.018 \\
\hline \multicolumn{6}{|l|}{ FIGO Stage } \\
\hline$I+I I$ & 77 & $44(57.1)$ & $33(42.9)$ & & \\
\hline III-IV & 30 & $25(83.3)$ & $5(16.7)$ & 6.466 & 0.011 \\
\hline \multicolumn{6}{|l|}{ Lymph Node Metastasis } \\
\hline No & 78 & $45(57.7)$ & $33(42.3)$ & & \\
\hline Yes & 29 & $24(82.8)$ & $5(17.2)$ & 5.800 & 0.016 \\
\hline
\end{tabular}

Table 3 Correlation Between the Expression of Wnt5a and EMT in Endometrial Tissue

\begin{tabular}{|c|l|l|l|l|l|}
\hline $\begin{array}{l}\text { EMT- } \\
\text { Related } \\
\text { Parameters }\end{array}$ & $\begin{array}{l}\text { Total/ } \\
\mathbf{n}\end{array}$ & $\begin{array}{l}\text { Wnt5a } \\
(-)\end{array}$ & $\begin{array}{l}\text { Wnt5a } \\
(+)\end{array}$ & $\gamma$ & P-value \\
\hline $\begin{array}{l}\beta \text {-catenin } \\
(-)\end{array}$ & 22 & 9 & 13 & & \\
$(+)$ & 85 & 60 & 25 & -0.251 & 0.009 \\
\hline $\begin{array}{c}\text { E-cadherin } \\
(-)\end{array}$ & 73 & 53 & 20 & & \\
$(+)$ & 34 & 16 & 18 & 0.249 & 0.010 \\
\hline $\begin{array}{c}\text { EZH2 } \\
(-)\end{array}$ & 32 & 15 & 17 & & \\
$(+)$ & 75 & 54 & 21 & -0.240 & 0.013 \\
\hline
\end{tabular}

phenotypes with the ability to invade and migrate. In tumor cells, particularly solid tumors, EMT enables in situ infiltration and distant metastasis. ${ }^{13}$ Many studies have shown that $\beta$-catenin, E-cadherin, and EZH2, as EMT-related protein factors, play an important role in promoting or inhibiting EMT. Understanding the role of Wnt5a in the regulation of EMT by EA is of great significance for determining the targets involved in invasion and metastasis.

$\beta$-catenin is widely expressed in various tissues and cells. As a core member of the Wnt signaling pathway, $\beta$-catenin plays an important role in mediating intercellular signaling, tumor invasion, and metastasis. Translocation of $\beta$-catenin to the nucleus indicates abnormal activation of the $\mathrm{Wnt} / \beta$-catenin signaling pathway ${ }^{14}$ and induces key factors that promote EMT. Baldus et $\mathrm{al}^{15}$ reported that high $\beta$-catenin expression in colorectal cancer is associated with accelerated disease progression and poor prognosis. Additionally, Yang et $\mathrm{al}^{16}$ detected morphological and molecular changes associated with EMT and found that $\beta$-catenin-mediated Wnt signaling promotes EMT by inducing EMT-related proteins. These proteins play an important role in invasion, metastasis, and chemotherapy resistance in colorectal cancer. Similarly, our results showed that the positive $\beta$-catenin expression rate gradually increased during the malignant transformation of endometrial tissue and there is an obvious negative correlation with the expression of Wnt5a, indirectly indicating that activation of the classical Wnt signaling pathway promotes EMT.

In normal epithelial cells, high expression levels of E-cadherin can inhibit cancer by preventing $\beta$-catenin from entering the nuclei. E-cadherin is downregulated in many solid tumors, such as diffuse gastric cancer and lobular breast cancer. ${ }^{17,18}$ Moreover, Xing et al ${ }^{19}$ analyzed 4383 patients with gastric cancer and found that E-cadherin downregulation is associated with invasion depth, histological type, and metastasis. In agreement, we found that the positive expression rate of E-cadherin decreases gradually during the malignant transformation 
WNT5A

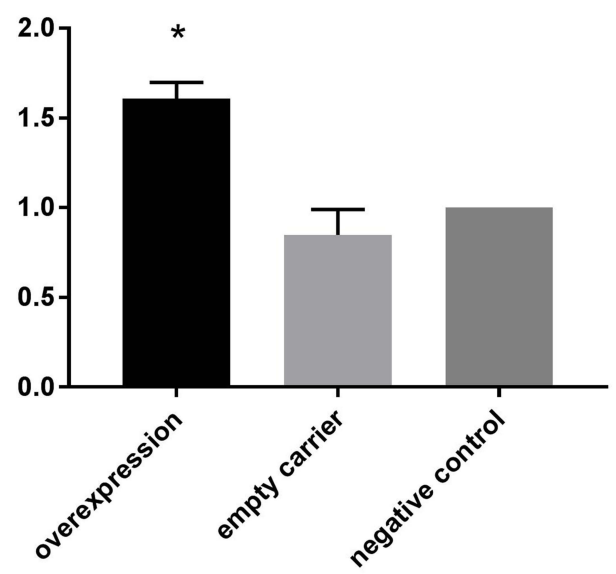

CDH1

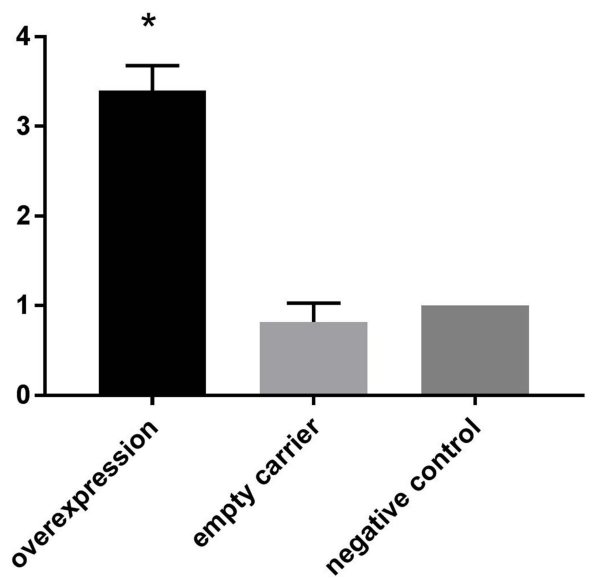

$E Z H 2$

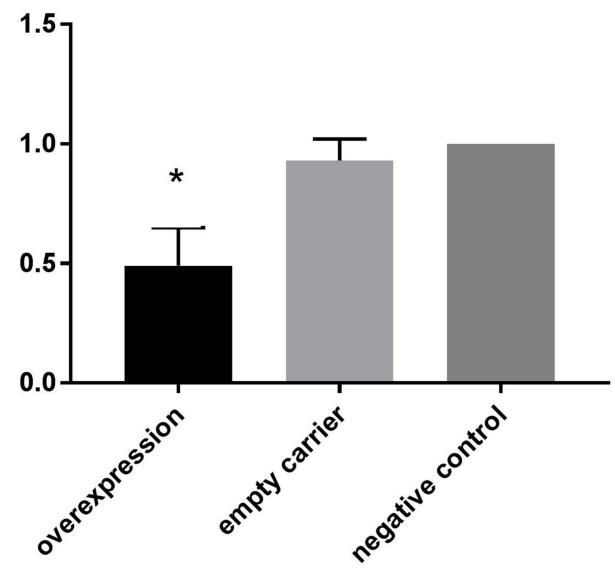

CTNNB1

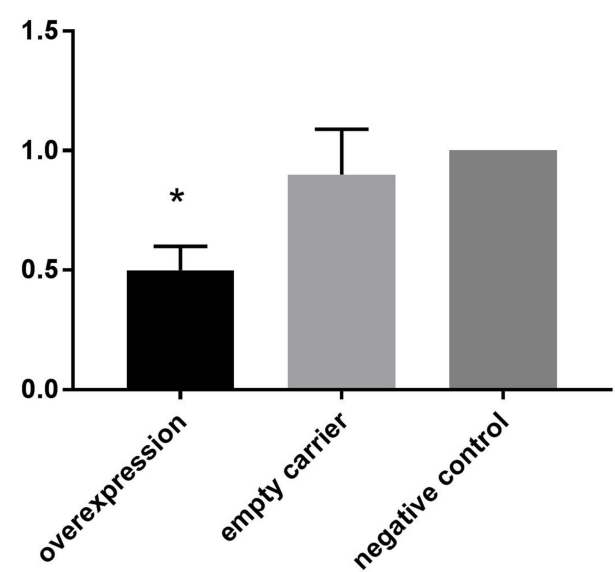

Figure 2 mRNA expression of WNT5A, EZH2, CDHI, and CTNNBI in endometrial carcinoma cell lines ( ${ }^{*}$ Indicates $P<0.01$ ).

of endometrial tissue, further suggesting that E-cadherin plays a role in the inhibition of EA. Furthermore, with an increase in the positive expression rate of E-cadherin, the positive expression rate of Wnt5a correspondingly increased, and there was a significant correlation between them $(P<0.05)$.

EZH2 in Drosophila is an important member of the multi-comb gene family and a transcription inhibitor and plays vital roles in cell differentiation, cell cycle regulation, and tumorigenesis. Kleer et $\mathrm{al}^{20}$ identified EZH2 as a molecular marker for invasive breast cancer and the tumorigenic transformation of breast epithelial cells, namely EMT. Similarly, Tonini et $\mathrm{al}^{21}$ showed that when $\mathrm{EZH} 2$ is overexpressed, the retention of histone deacetylase 1 activity related to $\mathrm{pRb} 2 / \mathrm{p} 130$ decreases substantially, leading to cell cycle progression and malignant transformation. In this study, the positive EZH2 expression rate increased gradually, whereas the positive Wnt5a expression rate decreased gradually during malignant transformation from normal endometrial tissue to endometrial tissue with atypical hyperplasia and endometrioid adenocarcinoma tissue. Moreover, there was a significant correlation between the expression levels of these two proteins $(P<0.05)$.

The Wnt signaling pathway is involved in EMT and promotes the accumulation of $\beta$-catenin in the nucleus. Moreover, Wnt signaling activates inhibitors of E-cadherin transcription. In this study, we found that during the malignant transformation of the endometrium, $\beta$-catenin and EZH2 levels increased as Wnt5a expression decreased, whereas E-cadherin levels decreased. Wnt5a expression was also found closely associated with the degree of tissue differentiation, FIGO stage, and lymph node metastasis. In addition, RT-PCR showed that the 


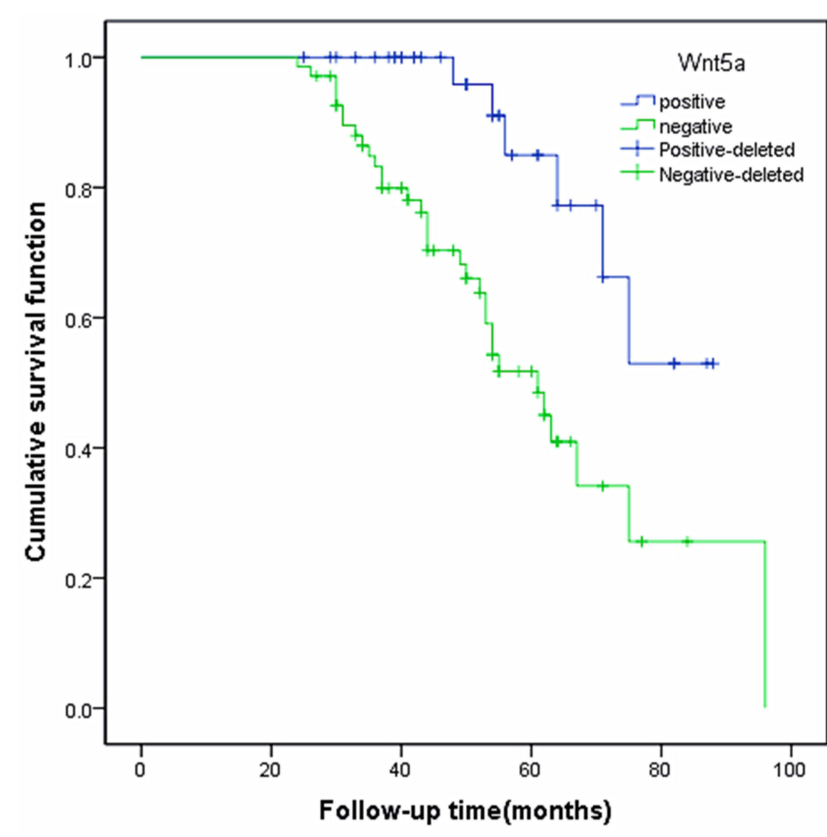

Figure 3 Relationship between Wnt5a expression and overall survival rate in patients with endometrioid adenocarcinoma.

expression of Wnt5a and E-cadherin in the Wnt5a overexpression group was significantly higher than that in the empty vector group and negative control group, but EZH2 and $\beta$-catenin were opposite. The results indicate that overexpression of Wnt5a can obviously inhibit the expression of EZH2 and $\beta$-catenin, and to some extent, inhibits or reverses the EMT process in endometrioid carcinoma.

\section{Conclusion}

The key role of Wnt5a, a non-classical Wnt ligand, in the Wnt signaling pathway is clearly established; however, few studies have evaluated the role of this protein in EMT. In this study, Wnt5a and EMT-related proteins were co-detected, revealing a correlation between their expression patterns and providing an experimental basis for further studies on the specific mechanism by which the Wnt signaling pathway influences EMT. In addition, the co-detection of these proteins may facilitate the accurate determination of EA pathogenesis and progression. Additional in-depth researches with larger sample sizes are needed to determine whether Wnt5a can be used as a molecular target for EA.

\section{Data Sharing Statement}

No additional data are available.

\section{Ethics Approval and Informed Consent}

Informed consent was obtained from all patients and their families. The study was approved by the Ethics Committee of the First Affiliated Hospital of Bengbu Medical College (NO. 2020057).

\section{Author Contributions}

NNY prepared the manuscript and contributed to data analysis and manuscript revision. $\mathrm{HCC}, \mathrm{YCH}$, and XXS assisted in cytological and histological experiments and directly participated in the revision of sections regarding the cytological experiments.. PPY, SZ, and WTY participate in the collection and interpretation of clinical data, and provide writing assistance related to format and grammar. ZZF and NL guided the first draft and revised draft of the paper, made pathological diagnosis and supervised the study. All authors made a significant contribution to the work reported, whether that is in the conception, study design, execution, acquisition of data, analysis and interpretation, or in all these areas; took part in drafting, revising or critically reviewing the article; gave final approval of the version to be published; have agreed on the journal to which the article has been submitted; and agree to be accountable for all aspects of the work.

\section{Funding}

This study was supported by the Natural Science Foundation of Anhui Province (No.1908085MH275); the Key Project of Natural Science Foundation of Bengbu Medical College (BYKF1710); the Joint Science and Technology Project of Bengbu City-Bengbu Medical College (BYLK201812).

\section{Disclosure}

The authors report no conflicts of interest in this work.

\section{References}

1. Bray F, Ferlay J, Soerjomataram I, Siegel RL, Torre LA, Jemal A. Global cancer statistics 2018: GLOBOCAN estimates of incidence and mortality worldwide for 36 cancers in 185 countries. CA Cancer J Clin. 2018;68(6):394-424. doi:10.3322/caac.21492

2. Hussein YR, Soslow RA. Molecular insights into the classification of high-grade endometrial carcinoma. Pathology. 2018;50(2):151-161. doi:10.1016/j.pathol.2017.09.010

3. Clark CC, Cohen I, Eichstetter I, et al. Molecular cloning of the human proto-oncogene Wnt-5A and mapping of the gene (WNT5A) to chromosome 3p14-p21. Genomics. 1993;18(2):249-260. doi:10.1006/ geno. 1993.1463

4. Oishi I, Suzuki H, Onishi N, et al. The receptor tyrosine kinase Ror2 is involved in non-canonical Wnt5a/JNK signalling pathway. Genes Cells. 2003;8(7):645-654. doi:10.1046/j.1365-2443.2003.00662.x 
5. Topol L, Jiang X, Choi H, Garrett-Beal L, Carolan PJ, Yang Y. Wnt$5 \mathrm{a}$ inhibits the canonical Wnt pathway by promoting GSK-3-independent beta-catenin degradation. J Cell Biol. 2003;162 (5):899-908. doi:10.1083/jcb.200303158

6. Asem MS, Buechler S, Wates RB, Miller DL, Stack MS. Wnt5a signaling in cancer. Cancers. 2016;8(9):79-96. doi:10.3390/ cancers 8090079

7. Tao J, Shi L, Huang L, et al. EZH2 is involved in silencing of WNT5A during epithelial-mesenchymal transition of colon cancer cell line. J Cancer Res Clin Oncol. 2017;143(11):2211-2219. doi:10.1007/s00432-017-2479-2

8. Kurayoshi M, Oue N, Yamamoto H, et al. Expression of Wnt-5a is correlated with aggressiveness of gastric cancer by stimulating cell migration and invasion. Cancer Res. 2006;66(21):10439-10448. doi:10.1158/0008-5472.CAN-06-2359

9. Kremenevskaja N, von Wasielewski R, Rao AS, Schöfl C, Andersson T, Brabant G. Wnt-5a has tumor suppressor activity in thyroid carcinoma. Oncogene. 2005;24(13):2144-2154. doi:10.1038/ sj.onc. 1208370

10. Zeng R, Huang J, Zhong MZ, et al. Multiple roles of WNT5A in breast cancer. Med Sci Monit. 2016;22:5058-5067. doi:10.12659/ MSM.902022

11. Dejmek J, Dejmek A, Säfholm A, Sjölander A, Andersson T. Wnt-5a protein expression in primary dukes B colon cancers identifies a subgroup of patients with good prognosis. Cancer Res. 2005;65 (20):9142-9146. doi:10.1158/0008-5472.CAN-05-1710

12. Bakker ER, Das AM, Helvensteijn W, et al. Wnt5a promotes human colon cancer cell migration and invasion but does not augment intestinal tumorigenesis in Apc1638N mice. Carcinogenesis. 2013;34(11):2629-2638. doi:10.1093/carcin/bgt215

13. Scanlon CS, Van Tubergen EA, Inglehart RC, D'Silva NJ. Biomarkers of epithelial-mesenchymal transition in squamous cell carcinoma. J Dent Res. 2013;92(2):114-121. doi:10.1177/ 0022034512467352
14. Kim S, Xu X, Hecht A, Boyer TG. Mediator is a transducer of Wnt/ beta-catenin signaling. J Biol Chem. 2006;281(20):14066-14075. doi:10.1074/jbc.M602696200

15. Baldus SE, Mönig SP, Huxel S, et al. MUC1 and nuclear beta-catenin are coexpressed at the invasion front of colorectal carcinomas and are both correlated with tumor prognosis. Clin Cancer Res. 2004;10 (8):2790-2796. doi:10.1158/1078-0432.CCR-03-0163

16. Yang AD, Fan F, Camp ER, et al. Chronic oxaliplatin resistance induces epithelial-to-mesenchymal transition in colorectal cancer cell lines. Clin Cancer Res. 2006;12(14):4147-4153. doi:10.1158/ 1078-0432.CCR-06-0038

17. Carvalho J, van Grieken NC, Pereira PM, et al. Lack of microRNA-101 causes E-cadherin functional deregulation through EZH2 up-regulation in intestinal gastric cancer. $J$ Pathol. 2012;228 (1):31-44.

18. Weigelt B, Geyer FC, Natrajan R, et al. The molecular underpinning of lobular histological growth pattern: a genome-wide transcriptomic analysis of invasive lobular carcinomas and grade- and molecular subtype-matched invasive ductal carcinomas of no special type. J Pathol. 2010;220(1):45-57. doi:10.1002/path.2629

19. Xing X, Tang YB, Yuan G, et al. The prognostic value of E-cadherin in gastric cancer: a meta-analysis. Int $J$ Cancer. 2013;132 (11):2589-2596. doi:10.1002/ijc.27947

20. Kleer CG, Cao Q, Varambally S, et al. EZH2 is a marker of aggressive breast cancer and promotes neoplastic transformation of breast epithelial cells. Proc Natl Acad Sci $U$ S A. 2003;100 (20):11606-11611. doi:10.1073/pnas.1933744100

21. Tonini T, Bagella L, D’Andrilli G, Claudio PP, Giordano A. Ezh2 reduces the ability of HDAC1-dependent $\mathrm{pRb} 2 / \mathrm{p} 130$ transcriptional repression of cyclin A. Oncogene. 2004;23(28):4930-4937. doi:10.1038/sj.onc. 1207608
Cancer Management and Research

\section{Publish your work in this journal}

Cancer Management and Research is an international, peer-reviewed open access journal focusing on cancer research and the optimal use of preventative and integrated treatment interventions to achieve improved outcomes, enhanced survival and quality of life for the cancer patient.

\section{Dovepress}

The manuscript management system is completely online and includes a very quick and fair peer-review system, which is all easy to use. Visit http://www.dovepress.com/testimonials.php to read real quotes from published authors. 